\title{
Predictive coding and the strong thesis of cognitive penetrability
} (La codificación predictiva y la tesis fuerte de la penetrabilidad cognitiva)

\author{
Sergio Cermeño-Aínsa* \\ Universidad Autónoma de Barcelona
}

\begin{abstract}
In this paper, I discuss the strong thesis of cognitive penetrability (CPs), to wit, that the perceptual states $(\mathrm{P})$ of a subject $(\mathrm{S})$ are pervasively influenced, affected, or caused by cognitive factors (C) as expectations, memories, thoughts, goals, and so on, at all levels of perceptual processing. I argue that following the predictive coding models of perception (PC), the strong thesis of cognitive penetrability is to be expected.
\end{abstract}

KEYWORDS: cognitive penetrability; predictive coding; top-down effects; early vision.

RESUMEN: En este artículo discuto la tesis fuerte de la penetrabilidad cognitiva (CP), a saber, que los estados perceptivos $(P)$ de un sujeto $(S)$ están siempre influidos, afectados o causados por factores cognitivos (C) como expectativas, recuerdos, pensamientos, metas, etc., a todos los niveles del procesamiento perceptivo. Sostengo que asumiendo los modelos de codificación predictiva de la percepción (PC), la tesis fuerte de la penetrabilidad cognitiva es lo más esperable.

PALABRAS CLAVE: penetrabilidad cognitiva; codificación predictiva; efectos top-down; visión temprana.

\footnotetext{
* Correspondence to: Sergio Cermeño-Aínsa. Escuela de Doctorado de la UAB. Programa de Doctorado en Ciencia Cognitiva y Lenguaje. Universidad Autónoma de Barcelona (08193 Cerdanyola del Vallès-Barcelona) - elsergei@hotmail.com - https://orcid.org/0000-0002-95988386
}

How to cite: Cermeño-Aínsa, Sergio (2021). "Predictive coding and the strong thesis of cognitive penetrability»; Theoria. An International Journal for Theory, History and Foundations of Science, 36(3), 341-360. (https://doi.org/10.1387/theoria.21949). 


\section{Introduction}

Traditional accounts of perception hold that all the information necessary to determine a perceptual experience is localized within the perceptual system, other non-perceptual systems are alien to this information, and only once perception has completed its work is this information integrated with other higher systems. Despite the strong intuitions derived from this view, there exists a considerable body of recent literature in experimental psychology and cognitive neuroscience consistent with the idea that perceptual processes inevitably involve theoretical presuppositions. This is the general idea defended by supporters of cognitive penetrability (CP), in short, that information from cognitive systems (e.g., thinking, remembering, or desiring) is indispensable for perceiving the world. What is ultimately suggested is that the intuitive distinction between seeing and thinking is, in a sense, fictitious - there is no clear delimitation between what we perceive and what we intend, think, or desire.

Such a lack of differentiation could, however, render the CP phenomenon meaningless, after all, for information of a cognitive state $(C)$ to influence the information of a perceptual state $(\mathrm{P})$, there should be a minimal distinction between $(\mathrm{C})$ and $(\mathrm{P})$. The debate might, at this point, be at a dead end. To keep the debate alive, some researchers have proposed to discard the folk understanding of perception and cognition and appeal to differences in the roles of bottom-up and top-down effects. For example, in characterizing a psychological process (for example, a perceptual process), we can ask how much the process is driven by incoming information from external stimuli, and how much it is affected by prior representations already in place before the stimulus was encountered. The cognitive penetrability debate can, thus, be recaptured by asking about the extent of top-down and bottom-up influences on a perceptual process (see Shea, 2015). If we accept this step, what brain science teaches us is that top-down processing is widespread in the brain (Gilbert and $\mathrm{Li}, 2013$ ). A perceptual experience thus becomes the product of the appropriate combination of both, bottom-up incoming sensory information and top-down stored knowledge. This results in a novel picture of the perception/cognition relationship, where perception is seen as the constant interaction between the information entering our retinas, and our background knowledge registered (trough learning and memory) as a model of the world. This new approach provides an interesting and integrated view of how these aspects of the mind are related.

However, there is a sense in which perception, understood as pure perception, resists being removed; it can still be seen as the source and cause of perceptual judgments, or as the more intimate and confidential connection with the objects in the world, or even as the spontaneous acquisition of some kind of experiential given knowledge. In this paper, I argue that the classic idea of perception as a form of natural given knowledge becomes blurry and increasingly moves away from the empirical evidence. This recent evidence challenges our more profound intuitions, showing why we should abandon the sharp distinction between perception and cognition hidden in our folk psychology and embrace the idea that perception and cognition are rather intertwined, inseparable, and all along working together. There are several reasons to think in this way. First, because on the basis of psychological studies it is not possible to isolate anything that deserves to be called pure perception, since even if it existed, it would always be contaminated by perceptual judgement, perceptual memory or perceptual recognition, thus becoming inaccessible to psychologi- 
cal methodologies (see Masrour et al., 2015; Cermeño-Aínsa, 2020). Second, because although psychological studies do not conclusively solve the debate on the perception/cognition relationship, they may not be necessary either - the intertwined view might still be supported by neurological evidence (Cecchi, 2018; although see Firestone and Scholl, 2016). And third, because the recent predictive processing framework (PC), the idea that the brain continually constructs predictions about the world, not only fits perfectly with the view delivered by such empirical discoveries, but also with the idea that perception cannot be isolated from cognition (Clark, 2013; Hohwy, 2013; see MacPherson, 2017 for a discussion about the relationship between PC and CP). What these reasons show, is that if we avoid postulating inaccessible substances or mediums between us and the world, however intuitive and intimate they may seem, and we pay attention to the most recent neurological findings and the tenets postulated by $\mathrm{PC}$, then the traditional view cannot stand any longer.

In this paper, I argue for the strong thesis of cognitive penetrability (CPs). CPs is, in fact, doubly strong, since argues that, first, all visual processing, also early vision, is cognitively penetrated (the all-levels claim), and second, that this occurs pervasively (the pervasiveness claim). Section 2 presents some controversies around what is usually understood by CP, putting emphasis on the cognitive penetration of early vision, traditionally (and functionally) defined by Pylyshyn $(1999,2003)$ and recently (temporally) redefined by Raftopoulos $(2009,2014,2017)$. I introduce, in section 3, a brief exposition of the predictive coding framework (PC). Section 4, argues for the all-levels claim. I first offer compelling evidence for very early top-down effects on perception, and subsequently, I focus on evidence from PC. I argue that the top-down predictive signal reaches the typical processes carried out during early vision and that the temporal criterion proposed by Raftopoulos is therefore out of place. Finally, in section 5, I argue for the pervasiveness claim. I propose that from PC we should expect CP not only at all levels but also pervasively. To the extent that sensory input is always uncertain, such uncertainty has to be minimized, and such minimization is mediated and modulated by top-down predictions, I argue that CP must be a ubiquitous phenomenon. Section 6 , summarizes my view.

\section{What is cognitive penetration?}

The general idea behind the cognitive penetrability of perception (CP) is the influence of mental states like beliefs, intentions, or desires over sensory perception in some relevant way. ${ }^{1}$ There are two distinct phenomena that go under the name of CP. The first is the CP of early vision. Early vision is defined functionally as the part of the visual system that takes signals from the eyes as inputs and produces shape, size, and colour representations as output (Pylyshyn, 1999)..$^{2}$ The other is the CP of perceptual experience. Perceptual experi-

1 In general, relevant cases of CP are those that produce epistemological consequences for the subjects. More specifically, are those that have consequences for the theory-ladenness of empirical observation, the epistemic role of perception, or (modular) architectures of the mind (see Stokes, 2015).

2 Importantly, Raftopoulos differentiates early and late vision by focusing on neuroscientific work, particularly, on the timing of neural processes in the visual system. Early vision is a pre-attentional stage of vision which includes a feed forward sweep (FFS) in which signals are transmitted bottom-up and which 
ence refers to the conscious phenomenal state that we typically go into when we perceive the world. The difference between early vision and visual experience is that the contents of visual experience are not exhausted by the contents of early vision so that the cognitive penetrability of perceptual experience is compatible with the cognitive impenetrability of early vision. In this paper, I will refer mostly to early vision.

As expected, philosophers disagree on how to define CP. For example, Pylyshyn (1999), who restricts CP to early vision, considers a system to be cognitively penetrable when

the function it computes is sensitive, in a semantically coherent way, to the organism's goals and beliefs, that is, it can be altered in a way that bears some logical relation to what the person knows. (Pylyshyn, 1999, p. 343)

This definition highlights the need for an intelligible link between the content of the cognitive state and the content of early vision, but neglects the type of causal relation between these contents (Stokes, 2013, 650; MacPherson, 2017, p. 10). MacPherson (2015), for example, provides the following case where the semantic condition is not sufficient for CP:

[S] uppose that Murdo believes that aliens are attacking Earth. This belief causes stress, which induces a migraine. Suppose that whenever Murdo has a migraine, he experiences flashing lights in the top half of his visual field. So, suppose that Murdo visually experiences flashing lights in the sky on account of having his migraine. The content of this experience bears a semantic relation to the belief that caused it - the belief about the alien attack - but one might want to deny that this is a case of cognitive penetration. (MacPherson, 2015, footnote 9)

These cases show the insufficiency of the semantic criterion, because even existing a semantic connection between the content of a cognitive state and the content of the perceptual experience, this is an accidental connection. Consequently, for CP to occur, the link between cognition and perception must be transferred from a cognitive state, so that its content has to be consistent, in a minimal rational sense, with the perceptual state, but there must also be a non-accidental causal semantic link at each stage of the process.

Consider now the following contrafactual formulation based in the necessary (and taken together sufficient) conditions for CP to occur: perception will be cognitively penetrable if it is possible for two subjects, or one subject at different times, to have different perceptual experience when the visual scene, the perceptual conditions, the spatial attention and the sensory organs, remain fixed (adapted from MacPherson, 2012, p. 29). It is to be expected that, maintaining a rational non-accidental connection between the cognitive and the perceptual states, the difference in their perceptual experiences must be caused

lasts, in visual areas, for about $100 \mathrm{~ms}$, and a stage at which lateral and recurrent connections allow recurrent processing (LRP). This sort of recurrent processing, which starts at about $80-100 \mathrm{~ms}$, is restricted within visual areas and does not involve signals from higher cognitive centers (Raftopoulos, 2014, 3). Late vision is, on the other hand, a stage modulated by attention in which visual processing and cognitive effects coexist. It is a hybrid stage of vision/thought in which perception and cognition are intermingled (Raftopoulos, 2011, 11). According to Raftopoulos early vision is cognitively impenetrable, whereas late vision is not. 
by differences in their cognitive states. ${ }^{3} \mathrm{~A}$ direct problem to these definitions is that focus on perceptual experience rather than early vision, so even meeting all these conditions, it is possible the $\mathrm{CP}$ of perceptual experience but not of early vision. Furthermore, the requirement of keeping the internal state of the sensory system fixed is in practice not very credible, the visual system needs to continually adapt, so demanding internal function to be fixed is an idealization that prevents the system to produce its internal changes.

Stokes (2013), on the other hand, downplays the semantic criterion and focuses his definition on the causal link. Indeed, he appreciates certain difficulties in the semantic criterion, but more than its sufficiency (as MacPherson does) he has doubts about its necessity. He postulates that there can be cases where background cognitive states affect the perceptual experience in a wholly semantically incoherent way, but nonetheless count as a real CP case (see Stokes, 2015, pp. 78-80). This possibility encourages Stokes to provide an alternative definition without appealing to normative considerations. He suggests the following definition

a perceptual experience $\mathrm{E}$ is cognitively penetrable if and only if (1) $\mathrm{E}$ is causally dependent on some cognitive state $\mathrm{C}$, and (2) the causal link between $\mathrm{E}$ and $\mathrm{C}$ is internal and mental. (Stokes, $2013,650)$

Clause (1) ensures that the relationship is direct, and clause (2) that it is internal. The causal chain must involve mental states or processes, thus ensuring that instances where intermediate states cause changes in perceptual experience do not count as CP. Stokes himself, however, doubts about the sufficiency of his own definition. Consider the following example: "Suppose I suffer extreme exam anxiety and I believe that I am about to take an exam. This belief causes, internally, another mental state, namely the pain that accompanies a migraine. This pain further causes, again internally, a series of visual experiences where everything appears in a reddish hue" (Stokes, 2013, 650). ${ }^{4}$ Both causal conditions are satisfied, but clearly, this is not a case of CP.

Finally, Stokes proposes a consequentialist understanding of CP (Stokes, 2015). According to this proposal, a definition of $\mathrm{CP}$ should be constrained by its consequences, so it will be successful just in case that it describes a phenomenon that has implications, says Stokes, for the theory-ladenness of perceptual observation, or for the epistemic role of perception, or for the modular theories of mental architecture. Thus, to be an instance of CP, at least, the following conditions must be fulfilled:

- There should be a genuine cognitive-perceptual relation (being this semantic, rational, causal, internal or whatever).

- And such relation should challenge the epistemic role of perception in justifying beliefs, or should grant that our empirical observations are theory-ladenness, or should challenge our current theories on mental architecture.

3 Siegel (2012) provides a similar formulation: "If visual experience is cognitively penetrable, then it is nomologically possible for two subjects (or for one subject in different counterfactual circumstances, or at different times) to have visual experiences with different contents while seeing and attending to the same distal stimuli under the same external conditions, as a result of differences in other cognitive (including affective) states" (Siegel 2012, 205-206).

4 The example is an adaptation of MacPherson $(2012,26)$, and it is originally used for emphasizing the necessity of the semantic criterion. 
These conditions move away from the discussions on the type of link expected between perception and cognition but emphasize both, the intervention of genuinely cognitive states during perceptual processing, as well as the implications of such an intervention for the theory-ladenness of perception or the epistemic role of perception or mental architecture. I think this better captures the essence of what $\mathrm{CP}$ must be, and can serve as an operational formulation. Yet, I insist, there is no consensus in the application of such definitions (mainly regarding the type of connection that would be expected between perception and cognition), like many other mental phenomena, finding a non-debatable definition becomes a very hard mission (for more profound and detailed discussions on the definitional concerns see Stokes, 2013, 2015; Macpherson, 2012, 2015).

Thus, setting aside the definitional controversies, a crucial aspect for this paper is to situate the locus and the scope of the $\mathrm{CP}$ phenomenon. For example, considering Raftopoulos or Pylyshyn accounts, being early vision the output of a perceptual encapsulated module, the contents of visual experience are not exhausted by the outputs of early vision, and therefore the cognitive penetrability of perceptual experience is compatible with the cognitive impenetrability of early vision (Raftopoulos, 2011). It is, therefore, possible to appreciate three different theses as to whether $\mathrm{CP}$ involves all, none, or only some parts of perceptual states. I call the strong cognitive penetrability thesis (CPs) the following statement:

(CPs) The perceptual states $(\mathrm{P})$ of a subject $(\mathrm{S})$ are pervasively influenced, affected, or caused by cognitive factors $(\mathrm{C})$ as expectations, memories, thoughts, goals, and so on, at all levels of perceptual processing.

To put it differently, there can be no perceptual states without minimal influence of cognitive states at all perceptual levels. ${ }^{5}$ Note that CPs is doubly strong. On the one hand, it entails the influence of cognitive factors at all levels of the visual process (the all-levels claim), and on the other, it postulates that $\mathrm{CP}$ is a pervasive phenomenon (the pervasive claim). Regarding the first claim, CPs is strong in contrast to the weak penetrability thesis $(\mathrm{CPw})$, which holds that only a part (late vision but not early vision) of the perceptual states of a subject is influenced by cognitive factors, an idea defended by Pylyshyn (1999) and more recently by Raftopoulos $(2009,2014)$. CPs is also opposite to the total impenetrability supporters, who hold that the perceptual states of a subject are not influenced by cognitive factors at any level, that is, that all the processes forming our perceptual experience are encapsulated from cognition, idea recently advocated by Firestone and Scholl (2016). CPs supports, then, that perception is cognitively penetrated at all levels, cognition affects perception at early and late levels of visual processing. Since I defend the CPs thesis, all my efforts will be concentrated on diminishing $\mathrm{CPw}$, which I think is the unique redoubt that remains to impenetrability supporters. Finally, and regarding the second claim, CPs states that cognition always and systematically influences perception, there cannot be perceptual

5 In this paper, I focus preferably on vision, mainly because it is a very well-studied field that provides very rich empirical data, but also because it is usually considered that all we can say about vision may be extended to other sense modalities. The process of vision or visual perception begins with the computation of a physical stimulus from the retina and finishes with the generation of a conscious or unconscious perceptual experience. 
states without a minimal influence of cognitive states. This is a very radical claim, there are in fact many proponents of the existence of CP, but few argue that CP is a pervasive phenomenon. As we will see pervasiveness arises as a natural consequence of embracing the predictive coding account of perception.

\section{The predictive coding account of perception}

The idea behind PC is that the brain produces top-down generative models or representations of the world that constitute predictions of how the world is (Rao and Ballard, 1999; Lee and Mumford, 2003; Hohwy, 2013; Clark, 2013; Friston, 2010). It is said that these top-down predictions facilitate perceptual processing by reducing the need to reconstruct the environment via exhaustive bottom-up analysis of incoming sensory information (Panichello et al., 2013, 4). Accordingly, the perceptual system relies on representations of prior probabilities to adjust the incoming sensory inputs to the representations stored in memory. Perceiving the world consists, therefore, in constantly elaborating estimations about how the world is, or more succinctly, perceiving the world is to balance the neuronal wiring configured in the brain with the cues arriving from the environment.

The brain is, therefore, constantly active. Cognitive and sensory systems work altogether interactively and concurrently, lower visual brain areas do not merely filter or extract environmental information, but participate as much as necessary along with the higher-levels in order to predict the incoming sensory stimulus. So, visual representations do not progress in a merely bottom-up serial fashion but occur interactively in constant feedforward and feedback loops that involve at the same time the entire hierarchical circuit in the visual system. According to this perspective rather than waiting to be activated by external influences, the brain is constantly generating expectations and predictions about the immediate future. To put simply, brains are essentially prediction machines (Clark, 2013).

The minimal version of $\mathrm{PC}^{6}$ is committed with the idea that the brain produces topdown generative representations that are bottom-up modified by incoming signals, these signals are propagated upwards only as a prediction error signal, and subsequently modified by a cascade of top-down processing, and perhaps sideways by other sensory modalities, to reduce global prediction error. Subsequent feedforward-feedback passes refine this process until the entire system settles on the most likely interpretation. The processing is typically thought to be done according to Bayesian rules (Clark, 2013; Hohwy, 2013; Lupyan, 2015; see also Macpherson, 2017, p. 10).

PC accounts of perception are sustained by a good number of instances. Rao and Ballard (1999), for example, provide a robust mathematical framework showing how hierarchical PC approach to perception describes how the sensory cortex extracts information from noisy stimuli. In this model of visual processing feedback connections from higher-order visual cortical areas to lower-order ones carry predictions of lower-level neural activi-

6 I say minimal because PC is not a unified framework, there is significant disagreement on the explanatory scope of the theory. The range of such an explanation goes from some neurocognitive functions to all the biological self-organization (Sims, 2017, 4). I am referring here to the minimal version. 
ties, whereas the feedforward connections carry the residual errors between the predictions and the actual lower-level activities. Hohwy et al. (2008) have shown that many aspects of the binocular rivalry phenomenon may be explained and unified by the predictive coding model. Panichello et al. (2013) have proposed that predictions enable rapid generation of conscious percepts and bias the contents of awareness in situations of uncertainty (for a review see Clark, 2013). Exploring this literature more scrupulously is beyond the scope of this paper, but increasingly, researchers find that from this attractive point of view a unified account of action, perception and cognition is possible.?

One can easily argue that this revolutionary idea runs in favour of CP: after all, once perception is understood as an inferential predictive process, perceptual systems are penetrable to the extent that such penetration minimizes global prediction error. If the information from non-perceptual systems (prior experience, expectations, knowledge, beliefs, etc.) influence perceptual processing by lowering global prediction error, then this information will be used to guide processing at lower levels (Lupyan, 2015). However, the relationship between PC and CP is currently under pressure: some theorists raise doubts about the real cognitive character of predictions (MacPherson, 2017, pp. 11-13), others doubt whether these predictions really affects the earlier states of perceptual processing (Raftopoulos, 2017, 975), others argue that in its current state of development PC cannot uncontrovertibly explain some interesting cases of CP (Vance and Stokes, 2017), and others argue that PC suffers from unsolvable difficulties such as the so-called dark room problem (Sun and Firestone, 2020). ${ }^{8}$

Summarizing, the PC account is not a fully accepted but a very plausible vision about how the mind/brain works. Recent disputes are, in fact, more about the scope of the thesis rather than its plausibility. Given its simplicity, elegance and explanatory scope, it seems that PC thesis is here to stay, we can be more precise in the mechanisms that underlie the process, but everything suggests that something must be right in the claim that our brains predict the incoming sensory stimulation. So, bearing all this in mind, let me analyse what the PC account teaches us about the relationship between perception and cognition, and more precisely, how PC leads to CPs.

7 PC is also consistent with other sense modalities, audition (Wacongne et al. (2012), taste (Gardner \& Fontanini, 2014), smell (Howard, Gottfried, Tobler, \& Kahnt, 2015) and touch (Naeije et al., 2016), as well as with other mental phenomena like theory of mind (Koster-Hale \& Saxe, 2013), mirror neurons (Kilner, Friston, \& Frith, 2007), attention (Feldman \& Friston, 2010), emotion (Seth, 2013), aesthetics (Cruys \& Wagemans, 2011), self-awareness (Apps \& Tsakiris, 2014), consciousness (Seth, Suzuki, \& Critchley, 2011), and language (Lupyan \& Clark, 2015). And it has also proved to be useful in explaining many symptoms associated to syndromes like schizophrenia (Fletcher \& Frith, 2009; Lalanne, van Assche, \& Giersch, 2010), psychosis (Corlett, Honey, \& Fletcher, 2016) and autism (Pellicano \& Burr, 2012).

8 The dark-room problem can be formulated as follows: if perception, action, and cognition are driven by the error minimization then why do not cognitive creatures act to minimize stimuli in general? Why cognitive creatures do not take up position in the nearest "dark room" and never move again? For some interesting responses to this concern see Friston, Thornton \& Clark (2012) and Cruys, Friston \& Clark (2020). 


\section{From predictive coding to the all-levels claim}

In section 2, I noted that there is a fundamental difference between the CP of perceptual experience and the CP of early vision. It may be the case that perceptual experience is cognitively penetrated while early vision is not. It is, in fact, very hard to determine what exactly a perceptual experience is, but what seems evident is that early vision is a perceptual process that leads to perceptual experience, that is, early vision is part of perceptual experience. There is a good number of examples showing the $\mathrm{CP}$ of perceptual experience (e.g., Levin \& Banaji, 2006; Balcetis \& Dunning, 2010; Stefanucci et al., 2012 to cite just a few; see however Firestone and Scholl, 2016), but whether there is CP of early vision is currently in the core of the debate. In this section, I assume the CP of perceptual experience, and review some evidence arguing that from PC the CP of early vision is to be expected (for further details see Vetter \& Newen, 2014, and specially Newen \& Vetter, 2017).

Cognitive scientists and neuroscientists have indeed put forward evidence for very early top-down effects on perception. For example, there is evidence showing top-down processing from motion area V5 to primary visual cortex V1 during motion perception (Silvanto, et al., 2005; Vetter et al., 2015), or top-down processing from the frontal eye fields (FEF), a higher-level area in frontal cortex involved in motor planning of eye movements, to V5 (Silvanto, et al., 2006; Morishima et al., 2009), and very early processing of object recognition (Drewes et al., 2016). ${ }^{9}$ Prominently, Potter et al. (2014) registered ultra-rapid object categorization as fast as $13 \mathrm{~ms}$. from stimulus onset, so far from the time interval suggested by Raftopoulos for early vision.

Following another line of thought, an analogy can be made between perception and mental imagery. There is strong evidence supporting the idea that visual imagery and visual perception share the same mechanisms and cortical regions (Kosslyn et al., 2006; Reddy et al., 2010). When one visually imagines an object, a kind of endogenous conceptual content is top-down elicited to construct a blurred visual representation of such object (including, of course, the low-level properties of the imagined visual stimulus). These top-down signals trigger activity within the early visual system. So, if such top-down signals can be elicited through imagination (endogenously) to produce representations within the visual system, why can't those same signals influence bottom-up processing in cases where vision occurs exogenously? In short, if visual imagery involves top-down activity over early visual brain areas, then there are no reasons to think that the same effects cannot be triggered during actual visual perception. I think that this line of thought may provide an excellent empirical contribution in support of the CP of early vision (see Ogilvie and Carruthers, 2015, pp. 728-730). ${ }^{10}$

In my view, all the above evidence is enough to accept the all-levels claim. However, in this paper I focus on evidence from PC. Since early vision is defined both functionally (by

9 For a more subtle description of these studies see Newen and Vetter (2017), for discussion see Raftopoulos (2017), and for further evidence of CP of early vision see O'Callaghan et al. (2017).

${ }^{10}$ Interestingly, Cecchi (2018) has also provided solid reasons and compelling examples to demonstrate that neural reorganization in the visual system is genuinely produced by CP. Other studies show that emotion enhances contrast sensitivity irrespective to attention, and potentiates the effect of attention on contrast sensitivity (Phelps, Ling, \& Carrasco, 2006), and others that emotional valence (e.g., happiness) of the stimuli can also facilitate the processing of biological motion (Lee \& Kim, 2017), 
Pylyshyn) and temporally (by Raftopoulos), it becomes pertinent to address these two possibilities. So, from PC one should empirically assess the CP of early vision in two ways, or by observing whether the top-down predictive signal reaches the typical processes carried out during early vision, or by analysing the temporal occurring of predictive and incoming signals and measure the temporal lapse between them.

Let me point out a number of examples of the first possibility. Top-down influences of prior knowledge affect colour processing (a typical dimension of early vision), most likely by constraining the inferences that the visual, system makes at earliest processing stages during complex natural scenes (Bannert and Bartels, 2013). Prior knowledge and expectations about the world also prepare perception by biasing incoming sensory information and influencing early visual responses (Samaha, Boutonnet, \& Lupyan, 2016). Shape perception (the grouping of local elements such as edges and lines into coherent shapes) is also modulated by predictive feedback, enhancing or reducing the activity in V1 depending on whether or not the predictive feedback meets with congruent bottom-up input (Kok and de Lange, 2014). Along with biased-competition, PC can also simulate a wide range of V1 response properties, including orientation tuning, size tuning, spatial frequency tuning, temporal frequency tuning, cross-orientation suppression and surround suppression (Spratling, 2010, 2011, 2012). Predictive signals can explain gain modulation as is observed when a retinal receptive field is modulated by eye position (De Meyer and Spratling, 2011, 2013), enhance the perceived contrast (Han and VanRullen, 2016), modulate contour integration (Spratling, 2013, 2014), or improve the detection of apparent motion (Vetter et al., 2012). These examples show that the pairing between top-down and bottom-up processes occur so early that even perceptual organization is subject to descending cognitive effects, thus showing that our prior knowledge, our goals or expectations may act over the structural organization of the stimuli. After all, it does not sound strange that our prior beliefs make us perceive the stimuli more vividly, more clearly, oriented in different ways or even sized, shaped and coloured differently in different circumstances or different situations. So, this being the case, even the determination of the structural descriptions of objects is subject to be altered by cognitive factors. So, regarding its functional definition, when viewing early vision as the system that produces shape, size and colour as representations, there exist compelling evidence showing that predictive feedbacks directly affect these functional (psychophysical) representations. Thus, we can conclude on the basis of predictive feedbacks that there is no room for the impenetrability of early vision (as defined functionally).

Let me now evaluate things from the point of view of the temporal criterion. The minimal version of PC postulates that perceptual inputs driven by stored knowledge form a sensory prediction of what we will see in the next time step. If that is right, human brains are representational models of the world, and with the model in hand, the brain extracts the information by continually seeking to reduce the prediction error. The information is extracted from sensory energy by predicting the input. Consequently, if such a prediction counts as the cognitive process influencing perception, the idea of a temporal space during which cognition does not influence perception loses its meaning because prediction precedes, or at least runs simultaneously, with the early stages of perception. As the model is constantly active in our brains, and our brains are prepared all the time to extract the information based on prior expectations, then such prior expectations (and not just sensory input) help us make sense of our environments. In sum, if PC involves the prediction or 
the advance of what we are going to see, and that is just what counts as CP, then the temporal criterion is, in principle, out of place.

Predictive processes must therefore be carried out within the period of time considered by Raftopoulos for early vision. For example, there are studies suggesting that directed spatial attention produced by the effects of feature salience enhance responses in the lateral geniculate nucleus (LGN), indicating that activity in the LGN can be altered by topdown attentional goals (Poltoratski et al., 2017; Ling et al., 2015; Jehee and Ballard, 2009). In these cases, the incoming signal, the exclusively bottom-up processing, does not alone go beyond the subcortical structures (LGN), which means that when the stimulus enters the cerebral cortex, predictive recurrent processing is not only underway, but it has already reached V1, the gateway of the visual cortex. Taking into account that incoming signals arrive at LGN before reaching V1 and arrive at V1 at 40-60ms. from stimulus onset (Nowak and Bullier, 1997), the predictive signal must be top-down transmitted at least as fast as this. In sum, prior knowledge stored in our heads may influence the visual system even before that incoming stimulus enters cortical areas. Other studies reveal similar results. For example, analysing the influence of prior experience on face perception, Gamond, et al. (2011) found that prior knowledge links with incoming current sensory input as fast as $65 \mathrm{~ms}$. from stimulus onset. Analysing the transfer of predictive signals across saccades in an apparent motion paradigm, Vetter, Edwards and Muckli (2012) found that the detection advantage of predictable targets is detectable as early as 50-100 ms. after saccade offset, thus showing very early latencies of predictive feedbacks. Although the temporal profile of the process in which predictive signals shape the incoming sensory stimuli has to be more deeply addressed, all this literature is consistent with the idea that if we take the first $100 \mathrm{~ms}$. of processing to be early vision, high-level predictive signals reach down as low as this.

In conclusion, if we assume that, as PC suggests, when viewing a stimulus, bottom-up incoming signals and top-down descending signals emerge simultaneously, then at the very moment that the stimulus enters the retina, the brain is already forming predictive mental images to facilitate perception. ${ }^{11}$ Since these predictive codes use sensory inputs and stored knowledge to form a sensory prediction trough their constant interaction, there will not be room for static and passive bottom-up processing. Indeed, the dynamic character of our mental life, interacting with the dynamic character of the world, means that priors may drive or alter how a subsequent image is visually processed. After all, if we are immersed in a changing world with a changing mind, it is conceivable that our perceptual states are the product of a variable rate of previous belief and incoming sensory inputs. Simply put, mostly (though perhaps not exclusively) unconsciously, we predict the incoming sensory stimuli in order to make sense of the spatio-temporal sequentiality of the environment. In the same way, we consistently predict the next causal link in a sequence of events in, for ex-

11 As expected, if bottom-up and top-down signals start their respective paths at the same time, at some intermediate point in the hierarchy the signals must encounter one another. The encounter of the ascendant and descendant signals produces the prediction error minimization and perhaps originates the conscious experience through the interaction between stimulus-driven and cognitive-driven attention (although this last point is highly speculative). The key point is that bottom-up signals never work alone (for a similar view see Clark, 2019). 
ample, the trajectory of moving objects, the behaviour of people in certain circumstances, or whatever. ${ }^{12}$

Of course, careful consideration of how to interpret the PC framework in the CP debate is needed (see MacPherson, 2017). However, given the initial evidence and the fact that the model is supported by a number of well-conducted studies, it is sensible to predict that CP occurs even at the earliest levels of perceptual processing. The core idea is that CP is a form of prediction and prediction is a form of $\mathrm{CP}$, that prediction permeates the entire visual hierarchy, influencing all levels of processing, thus suggesting that the all-levels claim proposed by CPs is the most suitable account.

\section{From predictive coding to the pervasive claim}

What about the pervasive claim? By admitting the all-levels claim, we are only admitting that CP may occur at any level of visual processing, but this is consistent with the idea that $\mathrm{CP}$ is a very rare and uncommon phenomenon. The pervasiveness of CP is undoubtedly what makes it really strong. ${ }^{13}$ To account for the pervasiveness claim-that perception is always, and not rarely, determined by cognition-I resort to Hohwy (2017). Hohwy presents an account that ties top-down modulation to a variable learning rate in hierarchical Bayesian inference. The learning rate refers to the difference between the influence of prior knowledge (the prediction or the top-down processing) and the influence of current

12 Surprisingly, what this picture seems to suggest is that visual perception is a process in which visual imagination is involved to some extent. This is because both perception and imagination share the endogenous top-down generation of their own sensory input. Perception and imagination are therefore not separate processes - perceivers are imaginers too (for discussion see Kirchhoff, 2018). Perceiving is then understood as an inferred fantasy about what lies behind the veil of input (Paton et al., 2013, 222), or as a process of controlled hallucination (Clark 2013, 25).

13 It should be noted that hidden in the idea of the pervasiveness of CP there is incrusted a potential dissolution of the perception/cognition boundary. In effect, this point is currently under pressure (for review see Phillips, 2019), and it could certainly be an inevitable consequence of the pervasive claim. After all, if perception is always determined by cognition, it is hard to find the point where perception ends and cognition begins. Let us consider three options of drawing such a distinction. First, there is a clear and sharp distinction between perception and cognition (monism). Second, there are multiple and blurry ways to distinguish perception and cognition (pluralism). And third, there is no distinction at all (eliminativism). PC, by its very nature, rejects the monist position but it is compatible with pluralism or eliminativism. CP, on the other hand, requires a minimal perception/cognition distinction, that is, rejects eliminativism but it is compatible with monism and pluralism. The point at issue here is, then, that monism is incompatible with PC, and eliminativism is incompatible with CP, so that only some kind of pluralism may be compatible with both. In consequence, for PC being compatible with $\mathrm{CP}$ we should consider a non-fixed distinction between perception and cognition, an idea compatible with a continuum along which lies more or less cognitive and more or less perceptual states (Newen and Vetter, 2017). Only in this case is CP intelligible and able to connect to PC. So, the position to be considered is, perforce, that of pluralism. My suggestion is then that PC, as a scientifically based theory, can be related to CP only if we are pluralists with respect to the perception/cognition distinction-to be more precise, with the idea that perception and cognition lie on a continuum along which there can be more or less cognitive and more or less perceptual states. 
sensory input (the prediction error or the bottom-up processing) in perceptual processing. $\mathrm{CP}$ is in this case conceived in terms of prediction error minimization deviations from this rate. What emerges here is a picture in which $\mathrm{CP}$ is determined by the learning rate. The lower the learning rate, the more top-down modulation and the more prevalent the cognitive influence on perception (high CP). And conversely, the higher the learning rate, the less influence of top-down beliefs (the more certain that the prior hypothesis is correct) and therefore the less prevalence of the cognitive influence on perception (low CP). So, assuming that to perceptually construct the world we need to infer the causes of sensory inputs, and considering that the construction of such perceptual inference is continually regulated by a variable rate of uncertainty, it follows that top-down modulation of perception by prior belief is inevitable (Hohwy, 2017, p. 84) ${ }^{14} \mathrm{CP}$ is therefore ubiquitous, although the extent in which perception is modulated by prior beliefs is, of course, variable. The more uncertainty, the more inaccurate the bottom-up prediction error, the greater topdown modulation, and the more prominent the influence of cognition on perception. In contrast, the less uncertainty, the more accurate the bottom-up prediction error, and therefore, the less top-down modulation and less prominent the influence of cognition on perception. It is then to be expected that such uncertainty (whether high or low) is solved by high-cognitive processes, and the most important, as our predictions are done according to Bayesian inferences, there should always be a minimum of uncertainty, and therefore, there should always be some kind of cognitive process exerting influence on perception.

The argument for the pervasiveness of CP relies, therefore, on the truth of the following assumptions:

(1) there is always some uncertainty about the world in sensory input.

(2) such perceptual uncertainty needs to be minimized in order to make sense the world.

(3) such a minimization is mediated and modulated by top-down predictions.

(4) such mediation and modulation count as relevant CP.

Hardly anyone denies (1) and (2), these assumptions are relatively unproblematic. That sensory information alone underdetermines perceptual integrations, and such underdetermination requires the contribution of further information to perceptual integrations is here assumed (although see Orlandi, 2015). However, (3) and (4) are far from being commonly accepted. Whether perceptual uncertainty is widespread and mediated by top-down predictions (3) and whether the nature of these predictions is cognitive and consequently counts as relevant to CP (4), are of course the key questions.

There exists compelling neuroscientific evidence to accept (3). I have assumed that PC reveals an essential part of the perceptual mind functioning, basically that the mere bottom-up information is insufficient to make sense the world. This is indeed corroborated by neuroscientific research, which reveals that all areas of the visual pathway, except for the retina, are subject to top-down influences, and most importantly, also reveals that these

14 This is related to the idea that perceptual inference is the process by which incoming sensory data is assimilated into a prior model of the world, which ultimately means that no level of neuronal processing hierarchies can be a priori excluded from the perceptual process (see Kiefer, 2017). I thank an anonymous reviewer for bringing this point. 
top-down influences constitute a necessary step to visually perceive the world. The predictions elicited from high-level information to lower-level activity becomes relevant to consummate the functional properties of these areas (Gilbert and Li, 2013). ${ }^{15}$ Therefore, premise (3), that the minimization of perceptual uncertainty is modulated by top-down predictions, is neuroscientifically supported.

Assessing the truth of (4) is more complicated. By accepting (3) we are admitting not only that top-down predictions occur, but also that they are pervasive, yet are these predictions really cognitive in nature? After all, predictions are high in relation to early vision, but are enough high to count as cognitive? Of course, everything depends on what we understand by cognition, or better, on whether the notion of cognition considered by $\mathrm{CP}$ and $\mathrm{PC}$ are equivalent. At this point, one can consider a strong and a weak sense of cognition. The strong sense refers only to doxastic states, that is, propositional attitudes like beliefs or desires that are accessible to consciousness and inferentially integrated, the weak notion refers also to non-doxastic states like moods, emotions, types of personality, cognitive styles, education, learning or expectations, which are in principle non-accessible to subject's consciousness. I do not see any particular obstacles in accommodating predictions to both the strong and the weak sense. Simply stated, if PC is on the right track, the prediction will sit variably high in the hierarchy depending on the uncertainty or ambiguity of stimuli. For example, the belief that my friend is upset with me can make me perceive my friend's facial features as particularly irritated, or the desire to see shooting stars can cause motion in static stars. These are cases where belief and desire (strong cognition) modulate perception. One can argue that cases where uncertainty is solved by high-level beliefs are perhaps scarce, in normal situations we perceptually reconstruct the world without the need of employing high-level beliefs (in the doxastic sense). If we take the weak sense of cognition, however, cases are not so scarce, moods, emotions, types of personality, cognitive styles, education, learning, or expectations, mostly unconscious, commonly influence perception. For example, when tracking a particular face in a crowded classroom, the expectation that the next stimulus is a face will be strengthened (we will tend to see a noisy non-face stimulus as a face), or a negative emotional state (e.g. sadness or fear) may also influence our perceptual experience (e.g. perceiving hills to be steeper or colors as darker), or types of personality, cognitive styles and moods can make us perceive stimuli in more vividly, clearly, oriented in different ways or even sized, shaped and colored differently in different circumstances or different situations. The critic may object that in the weak sense the prediction is not high-level enough to capture the essence of $\mathrm{CP}$, but a cognitive state need not be accessible to consciousness and inferentially integrated to be considered a cognitive state, and many alleged cases of CP do not adhere to such a strong restriction. So, if we take the idea that cognition is not exhausted by propositional attitudes (some non-propositional mental states also count as cognition) and that the influence of these states on perceptual states produces epistemological consequences for subjects, then these non-propositional mental constituents would be compelling cases of CP.

15 Importantly, Gilbert and $\mathrm{Li}$ (2013) do not limit top-down influences to attention, usually excluded by strong impenetrability supporters as a real top-down effect on perception, but to a much broader range of functional roles, including perceptual task, object expectation, scene segmentation, efference copy, working memory, and the encoding and recall of learned information. 
Finally, the philosophical relevance of CP when the uncertainty is solved with too low top-down modulation (when the prediction error is maximized) becomes the pertinent issue. Whether this kind of CP always describes a phenomenon that has implications for the theory-ladenness of perceptual observation, or for the epistemic role of perception, or for modular theories of mental architecture, becomes an empirical issue that, I think, is worth considering in future research.

\section{Concluding remarks}

In this paper, I have argued that the perceptual states of a subject are always influenced, affected or caused, by cognitive factors as expectations, memories, thoughts, goals and so on, at all levels of perceptual processing-the strong claim of CP (CPs). CPs is sustained by two claims, the first is that CP occurs at all levels of perceptual processing (the all-levels claim), and the second that this influence occurs widespread (the pervasiveness claim). I have not discussed the CP of all the processes that make up our perceptual experience because I think that empirical evidence has amply demonstrated that in the construction of perceptual experiences cognitive processes are necessary. This ultimately means that at the experiential level a sharp boundary between perception and cognition is unsustainable. What it is currently at the core of the debate is, instead, the influence of cognitive states over early visual processes. I have argued that working within the constraints of the predictive coding theory of brain function, the two claims derived from CPs are to be expected.

To account for the all-levels claim I have considered two definitions of early vision, the functional and the temporal definitions. Regarding the functional definition, I have provided evidence suggesting that predictive feedbacks directly modulate the typical functional (psychophysical) properties represented during early vision. Regarding the temporal definition, I have argued that from the point of view of PC, the temporal criterion to sustain the cognitive impenetrability of early vision does not make sense, since bottom-up and top-down signals could begin their respective paths, at least, simultaneously. I have also reviewed empirical evidence suggesting that predictions are elicited within the first $100 \mathrm{~ms}$. from stimulus onset, thus showing that even the initial states of perceptual processing are influenced by cognitive factors. In sum, the all-levels claim is asserted.

To account for the pervasive claim, I have first assumed that sensory inputs always have some doses of uncertainty that must be minimized in order to make sense of the world. Subsequently, from neuroscientific research, I have shown that such minimization must be mediated and modulated by top-down predictions-top-down descending pathways are ubiquitous in the brain. Finally, I have argued that such predictions are cognitive in nature. Consequently, in the construction of perceptual experiences a minimal cognitive influence there should constantly be present. The pervasive claim is, therefore, sustained.

Thus, the sum of the evidence from psychological, neurological and behavioural empirical data in addition to the fact that it fits perfectly with PC accounts of perception, suggests that CPs is the most plausible account. 


\section{Acknowledgements}

Versions of this material were presented in a Seminary in the Autonomous University of Barcelona. I would like to thank those in attendance for their critical feedback, as well as to Olga Fernandez Prat for her useful comments and advise. I would also like to thank two anonymous referees for this journal, for their helpful comments on an earlier draft.

\section{REFERENCES}

Apps, M. A. J., \& Tsakiris, M. (2014). The free-energy self: A predictive coding account of self-recognition. Neuroscience and Biobehavioral Reviews, 41, 85-97. https://doi.org/10.1016/j.neubiorev.2013.01.029

Balcetis, E. \& Dunning, D. (2010). Wishful seeing: More desired objects are seen as closer. Psychological Science, 21, 147-52. https://doi.org/10.1177/0956797609356283

Bannert, M. M., \& Bartels, A. (2013). Decoding the yellow of a gray banana. Current Biology, 23(22), 22682272. https://doi.org/10.1016/j.cub.2013.09.016

Cecchi, A. (2018). Cognitive penetration of early vision in face perception. Consciousness and Cognition, 63, 254-266. https://doi.org/10.1016/j.concog.2018.06.005

Cermeño-Ainsa, S. (2020). The cognitive penetrability of perception: A blocked debate and a tentative solution. Consciousness and Cognition, 77, 102838. https://doi.org/10.1016/j.concog.2019.102838

Clark, A. (2013). Whatever next? Predictive brains, situated agents, and the future of cognitive science. Behavioural and Brain Sciences, 36(3), 181-204. https://doi.org/10.1017/S0140525X12000477

Clark, A. (2019). Consciousness as generative entanglement. The Journal of Philosophy, 116, 645-662. https://doi.org/10.5840/jphil20191161241

Corlett, P. R., Honey, G. D., \& Fletcher, P. C. (2016). Prediction error, ketamine and psychosis: An updated model. Journal of Psychopharmacology, 30(11), 1145-1155. https://doi. org $/ 10.1177 / 0269881116650087$

Cruys, S.V., \& Wagemans, J. (2011). Putting reward in art: A tentative prediction error account of visual art. i-Perception, 2, 1035-1062. https://doi.org/10.1068/i0466aap

Cruys, S.V., Friston, K. \& Clark, A. (2020). Controlled optimism: Reply to Sun and Firestone on the dark room problem. Trends in Cognitive Science, 24, 680-681. https://doi.org/10.1016/j.tics.2020.05.012

De Meyer, K. \& Spratling, M. W. (2011). Multiplicative gain modulation arises through unsupervised learning in a predictive coding model of cortical function. Neural Computation, 23(6), 1536-67. https://doi. org/10.1162/NECO_a_00130

De Meyer, K. \& Spratling, M. W. (2013). A model of partial reference frame transforms through pooling of gain-modulated responses. Cerebral Cortex, 23(5), 1230-9. https://doi.org/10.1093/cercor/bhs1 17

Drewes, J., Goren, G., Zhu, W., \& Elder, J. H. (2016). Recurrent processing in the formation of shape percepts. Journal of Neuroscience, 36(1), 185-192. https://doi.org/10.1523/JNEUROSCI.2347-15.2016

Feldman, H., \& Friston, K. J. (2010). Attention, uncertainty, and free-energy. Frontiers in Human Neuroscience, 4, 215. https://doi.org/10.3389/fnhum.2010.00215

Firestone, C. \& Scholl, B. J. (2016). Cognition does not affect perception: Evaluating the evidence for 'top-down' effects. Behavioural and Brain Sciences, 39, 1-77. https://doi.org/10.1017/ S0140525X15000965

Fletcher, P. C., \& Frith, C. D. (2009). Perceiving is believing: A Bayesian approach to explaining the positive symptoms of schizophrenia. Nature Reviews Neuroscience, 10, 48-58. https://doi.org/10.1038/nrn2536

Friston, K. J. (2010). The free-energy principle: A unified brain theory? Nature Reviews Neuroscience 11(2), 127-38. https://doi.org/10.1038/nrn2787

Friston, K., Thornton, C., \& Clark, A. (2012). Free-energy minimization and the dark-room problem. Frontiers in Psychology, 3, 130. https://doi.org/10.3389/fpsyg.2012.00130 
Gamond, L., George, N., Lemarechal, J.D. et al. (2011). Early influence of prior experience on face perception. Neuroimage, 54, 1415-26. https://doi.org/10.1016/j.neuroimage.2010.08.081

Gardner, M. P. H., \& Fontanini, A. (2014). Encoding and tracking of outcome-specific expectancy in the gustatory cortex of alert rats. Journal of Neuroscience, 34(39), 13000-13017. https://doi.org/10.1523/ JNEUROSCI.1820-14.2014

Gilbert, C. D., \& Li, W. (2013). Top-down influences on visual processing. Nature Reviews Neuroscience, 14, 350-363. https://doi.org/10.1038/nrn3476

Han, B., \& VanRullen, R. (2016). Shape perception enhances perceived contrast: evidence for excitatory predictive feedback? Scientific reports, 6, 22944. https://doi.org/10.1038/srep22944ç

Hohwy, J. (2013). The predictive mind. Oxford: Oxford University Press.

Hohwy, J. (2017). Priors in perception: Top-down modulation, Bayesian perceptual learning rate, and prediction error minimization. Consciousness and Cognition, 47, 75-85. https://doi.org/10.1016/j.concog.2016.09.004

Hohwy, J., Roepstorff, A. \& Friston, K. (2008). Predictive coding explains binocular rivalry: An epistemological review. Cognition, 108(3), 687-701. https://doi.org/10.1016/j.cognition.2008.05.010

Howard, J. D., Gottfried, J. A., Tobler, P. N., \& Kahnt, T. (2015). Identity-specific coding of future rewards in the human orbitofrontal cortex. Proceedings of the National Academy of Sciences, 112(16), 5195-5200. https://doi.org/10.1073/pnas.1503550112

Jehee, J. F. M. \& Ballard, D. H. (2009). Predictive feedback can account for biphasic responses in the lateral geniculate nucleus. PLoS Computational Biology 5(5), e1000373. https://doi.org/10.1371/journal. pcbi. 1000373

Kiefer, A. (2017). Literal Perceptual Inference. In T. Metzinger \& W. Wiese (Eds.). Philosophy and Predictive Processing, 17. Frankfurt am Main: MIND Group. https://doi.org/10.15502/9783958573185

Kilner, J. M., Friston, K. J., \& Frith, C. D. (2007). Predictive coding: An account of the mirror neuron system. Cognitive Processing, 8(3), 159-166. https://doi.org/10.1007/s10339-007-0170-2

Kirchhoff, M.D. (2018). Predictive processing, perceiving and imagining: Is to perceive to imagine, or something close to it? Philosophical Studies, 175(3), 751-767. https://doi.org/10.1007/s11098-017-0891-8

Kok, P., \& de Lange, F.P. (2014). Shape perception simultaneously up- and downregulates neural activity in the primary visual cortex. Current Biology, 24(13), 1531-1535. https://doi.org/10.1016/j. cub.2014.05.042

Kosslyn, S., W. Thompson, \& Ganis, G. (2006). The case for mental imagery. Oxford: Oxford University Press.

Koster-Hale, J., \& Saxe, R. (2013). Theory of mind: A neural prediction problem. Neuron, 79(5), 836-848. https://doi.org/10.1016/j.neuron.2013.08.020

Lalanne, L., van Assche, M., \& Giersch, A. (2010). When predictive mechanisms go wrong: Disordered visual synchrony thresholds in schizophrenia. Schizophrenia Bulletin, 38(3), 506-513. https://doi. org/10.1093/schbul/sbq107

Lee, H., \& Kim, J. (2017). Facilitating effects of emotion on the perception of biological motion: Evidence for a happiness superiority effect. Perception, 46(6), 679-697. https://doi. org/10.1177/0301006616681809

Lee, T.S., \& Mumford, D. (2003). Hierarchical Bayesian inference in the visual cortex. Journal of Optical Society of America, 20(7), 1434-1448. https://doi.org/10.1364/JOSAA.20.001434

Levin, D., \& Banaji, M. (2006). Distortions in the perceived lightness of faces: The role of race categories. Journal of Experimental Psychology, 135, 501-512. https://doi.org/10.1037/0096-3445.135.4.501

Ling, S., Pratte, M.S., \& Tong, F. (2015). Attention alters orientation processing in the human lateral geniculate nucleus. Nature Neuroscience, 18, 496-498. https://doi.org/10.1038/nn.3967

Lupyan, G. (2015). Cognitive penetrability of perception in the age of prediction: Predictive systems are penetrable systems. Review of Philosophy and Psychology, 6, 547-569. https://doi.org/10.1007/s13164015-0253-4 
Lupyan, G., \& Clark, A. (2015). Words and the world: Predictive coding and the language-perception-cognition interface. Current Directions in Psychological Science, 24(4), 279-284. https://doi. org/10.1177/0963721415570732

Macpherson, F. (2012). Cognitive penetration of colour experience: Rethinking the issue in light of an indirect mechanism. Philosophy and Phenomenological Research, 84(1), 24-62. https://doi.org/10.1111/ j.1933-1592.2010.00481.x

Macpherson, F. (2015). Cognitive penetration and predictive coding: A commentary on Lupyan. Review of Philosophy and Psychology, 6(4), 571-584. https://doi.org/10.1007/s13164-015-0254-3

Macpherson, F. (2017). The relationship between cognitive penetration and predictive coding. Consciousness and Cognition, 47: 6-16. https://doi.org/10.1016/j.concog.2016.04.001

Masrour, F., Nirshberg, G., Schon, M., Leardi, J. \& Barrett, E. (2015). Revisiting the empirical case against perceptual modularity. Frontiers in Psychology, 6, 1676. https://doi.org/10.3389/fpsyg.2015.01676

Morishima, Y., Akaishi, R., Yamada, Y., Okuda, J., Toma, K., \& Sakai, K. (2009). Task-specific signal transmission from prefrontal cortex in visual selective attention. Nature Neuroscience, 12(1), 85-91. https:// doi.org/10.1038/nn.2237

Naeije, G., Vaulet, T., Wens, V., Marty, B., Goldman, S., \& De Tiege, X. (2016). Multilevel cortical processing of somatosensory novelty: A magnetoencephalography study. Frontiers in Human Neuroscience, 10, 259. https://doi.org/10.3389/fnhum.2016.00259

Newen, A. \& Vetter, P. (2017). Why cognitive penetration of our perceptual experience is still the most plausible account. Consciousness and Cognition, 47, 26-37. https://doi.org/10.1016/j.concog.2016.09.005

Nowak, L., \& Bullier, J. (1997). The Timing of Information Transfer in the Visual System. In: Rockland K.S., Kaas J.H., Peters A. (eds.) Extrastriate Cortex in Primates. Cerebral Cortex, 12, (pp 205-241). Springer, Boston, MA. https://doi.org/10.1007/978-1-4757-9625-4_5

O’Callaghan, C., Kveraga, K., Shine, J. M., Adams, Jr. \& Bar, M. (2017). Predictions penetrate perception: Converging insights from brain, behaviour and disorder. Consciousness and Cognition, 47, 63-74. https://doi.org/10.1016/j.concog.2016.05.003

Ogilvie, R., \& Carruthers, P. (2016). Opening up vision: The Case against encapsulation. Review of Philosophy and Psychology, 7, 721-742. https://doi.org/10.1007/S13164-015-0294-8

Orlandi, N. (2016). Bayesian perception is ecological perception. Philosophical Topics, 44(2), 327-352. https://doi.org/10.5840/philtopics201644226

Panichello, M. F., Cheung, O. S. \& Bar, M. (2013). Predictive feedback and conscious visual experience. Frontiers in Psychology, 3(620), 1-8. https://doi.org/10.3389/fpsyg.2012.00620

Paton, B., Skewes, J., Frith, C., \& Hohwy, J. (2013). Skull-bound perception and precision optimization through culture. The Behavioural and Brain Sciences, 36(3), 42. https://doi.org/10.1017/ S0140525X12002191

Pellicano, E., \& Burr, D. (2012). When the world becomes 'too real': A Bayesian explanation of autistic perception. Trends in Cognitive Sciences, 16, 504-510. https://doi.org/10.1016/j.tics.2012.08.009

Phelps, E. A., Ling, S., \& Carrasco, M. (2006). Emotion facilitates perception and potentiates the perceptual benefits of attention. Psychological Science, 17(4), 292-299. https://doi.org/10.1111/j.14679280.2006.01701.x

Phillips, B. (2019). The shifting border between perception and cognition. Nồs, 53(2), 316-346. https:// doi.org/10.1111/nous.12218

Poltoratski, S., Ling, S., McCormack, D., \& Tong, F. (2017). Characterizing the effects of feature salience and top-down attention in the early visual system. Journal of Neurophysiology, 118, 564-573. https://doi. org/10.1152/jn.00924.2016

Potter, M., Wyble, B., Hagmann, C., \& McCourt, E. (2014). Detecting meaning in RSVP at 13 ms per picture. Attention, Perception \& Psychophysics, 76(2), 270-279. https://doi.org/10.3758/s13414-0130605-z 
Pylyshyn, Z. W. (1999). Is vision continuous with cognition? The case for cognitive impenetrability of visual perception. Behavioural and Brain Sciences, 22, 341-423. https://doi.org/10.1017/ S0140525X99002022

Pylyshyn, Z. W. (2003). Seeing and visualizing: It's not what you think. Cambridge, MA: MIT Press.

Raftopoulos, A. (2009). Cognition and perception: How do psychology and neural sciences inform philosophy? Cambridge, MA: MIT Press.

Raftopoulos, A. (2011). Late vision: Processes and epistemic status. Frontiers in Psychology, 2 (382), 1-12. https://doi.org/10.3389/fpsyg.2011.00382

Raftopoulos, A. (2014). The cognitive impenetrability of the content of early vision is a necessary and sufficient condition for purely nonconceptual content. Philosophical Psychology, 27(5), 601-620. https://doi. org/10.1080/09515089.2012.729486

Raftopoulos, A. (2017). Timing time: Why early vision is cognitively Impenetrable. Proceedings of the 39th Annual Conference of the Cognitive Science Society, 26-29 July (pp. 974-979). Austin, TX: Cognitive Science Society.

Rao, R. P. N. \& Ballard, D. H. (1999). Predictive coding in the visual cortex: A functional interpretation of some extra-classical receptive-field effects. Nature Neuroscience, 2(1), 79-87. https://doi. org/10.1038/4580

Samaha, J., Boutonnet, B., \& Lupyan, G. (2016). How prior knowledge prepares perception: Prestimulus oscillations carry perceptual expectations and influence early visual responses. bioRxiv. https://doi. org/10.1101/076687

Seth, A. K., Suzuki, K., \& Critchley, H. D. (2011). An interoceptive predictive coding model of conscious presence. Frontiers in Psychology, 2 (395). https://doi.org/10.3389/fpsyg.2011.00395

Seth, A. K. (2013). Interoceptive inference, emotion, and the embodied self. Trends in Cognitive Sciences, 17(11), 656-663. https://doi.org/10.1016/j.tics.2013.09.007

Shea, N. (2015). Distinguishing Top-Down from Bottom-Up Effects. In D. Stokes, M. Matthen \& S. Biggs (Eds.), Perception and its modalities (pp. 73-91). Oxford: Oxford University Press. https://doi. org/10.1093/acprof:oso/9780199832798.003.0004

Siegel, S. (2012). Cognitive penetrability and perceptual justification. Nồs, 46(2), 1-22. https://doi. org/10.1111/j.1468-0068.2010.00786.x

Silvanto, J., Lavie, N., \& Walsh, V. (2005). Double dissociation of V1 and V5/MT activity in visual awareness. Cerebral Cortex, 15, 1736-1741. https://doi.org/10.1093/cercor/bhi050

Silvanto, J., Lavie, N., \& Walsh, V. (2006). Stimulation of the human frontal eye fields modulates sensitivity of extrastriate visual cortex. Journal of Neurophysiology, 96(2), 941-945. https://doi.org/10.1152/ jn.00015.2006

Sims, A. (2017). The problems with prediction - The dark room problem and the scope dispute. In T. Metzinger \& W. Wiese (Eds.). Philosophy and Predictive Processing: 23. Frankfurt am Main: MIND Group. https://doi.org/10.15502/9783958573246

Spratling, M. W. (2010). Predictive coding as a model of response properties in cortical area V1. Journal of Neuroscience, 30(9), 3531-543. https://doi.org/10.1523/JNEUROSCI.4911-09.2010

Spratling, M. W. (2011). A single functional model accounts for the distinct properties of suppression in cortical area V1. Vision Research, 51(6), 563-76. https://doi.org/10.1016/j.visres.2011.01.017

Spratling, M. W. (2012). Predictive coding accounts for V1 response properties recorded using reverse correlation. Biological Cybernetics, 106(1), 37-49. https://doi.org/10.1007/s00422-012-0477-7

Spratling, M. W. (2013). Image segmentation using as parse coding model of cortical area V1. IEEE Transactions on Image Processing, 22(4), 1631-43. https://doi.org/10.1109/TIP.2012.2235850

Spratling, M. W. (2014). A single functional model of drivers and modulators in cortex. Journal of Computational Neuroscience, 36(1), 97-118. https://doi.org/10.1007/s10827-013-0471-7

Stefanucci, J. K., Gagnon, K. T., Tompkins, C. L. \& Bullock, K. E. (2012). Plunging into the pool of death: Imagining a dangerous outcome influences distance perception. Perception, 41, 1-11. https://doi. org/10.1068/p7131 
Stokes, D. (2013). Cognitive penetrability of perception. Philosophy Compass, 8(7), 646-663. https://doi. org/ $10.1111 /$ phc3.12043

Stokes, D. (2015). Towards a consequentialist understanding of cognitive penetration. In A. Raftopoulos and J. Ziembekis (Eds.), Cognitive Effects on Perception: New Philosophical Perspectives (pp. 75-100). Oxford: Oxford University Press.

Sun, Z. \& Firestone, C. (2020). The dark room problem. Trends in Cognitive Science, 24, 346-348. https:// doi.org/10.1016/j.tics.2020.02.006

Vance, J., \& Stokes, D. (2017). Noise, uncertainty, and interest: Predictive coding and cognitive penetration. Consciousness and Cognition, 47, 86-98. https://doi.org/10.1016/j.concog.2016.06.007

Vetter, P., \& Newen, A. (2014). Varieties of cognitive penetration in visual perception. Consciousness and Cognition, 27, 62-75. https://doi.org/10.1016/j.concog.2014.04.007

Vetter, P., Edwards, G., \& Muckli, L. (2012). Transfer of predictive signals across saccades. Frontiers in psychology, 3, 176. https://doi.org/10.3389/fpsyg.2012.00176

Vetter, P., Grosbras, M. H., \& Muckli, L. (2015). TMS over V5 disrupts motion prediction. Cerebral Cortex, 25(4), 1052-1059. https://doi.org/10.1093/cercor/bht297

Wacongne, C., Changeux, J. P. \& Dehaene, S. (2012). A neuronal model of predictive coding accounting for the mismatch negativity. Journal of Neuroscience 32, 3665-78. https://doi.org/10.1523/JNEUROSCI.5003-11.2012

SERGIO CERMEÑO-AÍNSA is a doctoral student enrolled in the Cognitive Science and Language (CCiL) program at the Autonomous University of Barcelona. He specializes in foundations of cognitive science, philosophy of mind and epistemology, in particular, topics related to perception and its relation to cognition. He has published a paper ("The Cognitive penetrability of perception: A blocked debate and a tentative solution") in a top rank journal (Consciousness and Cognition), and he also has three papers under review in top-ranking journals. In addition to the cognitive penetrability of perception and the perception/cognition distinction, he is currently interested in the mechanisms behind the phenomenon of implicit bias.

ADDRESS: Escuela de Doctorado de la UAB. Programa de Doctorado en Ciencia Cognitiva y Lenguaje. Universidad Autónoma de Barcelona, 08193 Cerdanyola del Vallès, Barcelona. E-mail: elsergei@hotmail.com ORCID: https://orcid.org/0000-0002-9598-8386 